\title{
CATÁLOGO PRELIMINAR DE LA FLORA VASCULAR DEL VALLE DEL RÍO TÉMPANO, MAGALLANES, CHILE.
}

\author{
PRELIMINAR CATALOGUE OF VASCULAR FLORA FROM THE \\ RIO TEMPANO VALLEY, MAGALLANES, CHILE.
}

Alejandra Silva ${ }^{1}$, Rina Charlín ${ }^{1} \&$ Orlando Dollenz ${ }^{2}$

La Corporación Nacional Forestal desarrolla un proyecto de conservación del huemul (Hippocamelus bisulcus) en el valle del río Témpano, 48 49' S - 74ํㅜㄹ' W (Fig.1, imagen Google Earth) ubicado en el archipiélago occidental de la región de Magallanes, en el límite con la región de Aisén. Parte de este proyecto contempla el estudio de la flora vascular y las formaciones vegetales existentes en el valle.

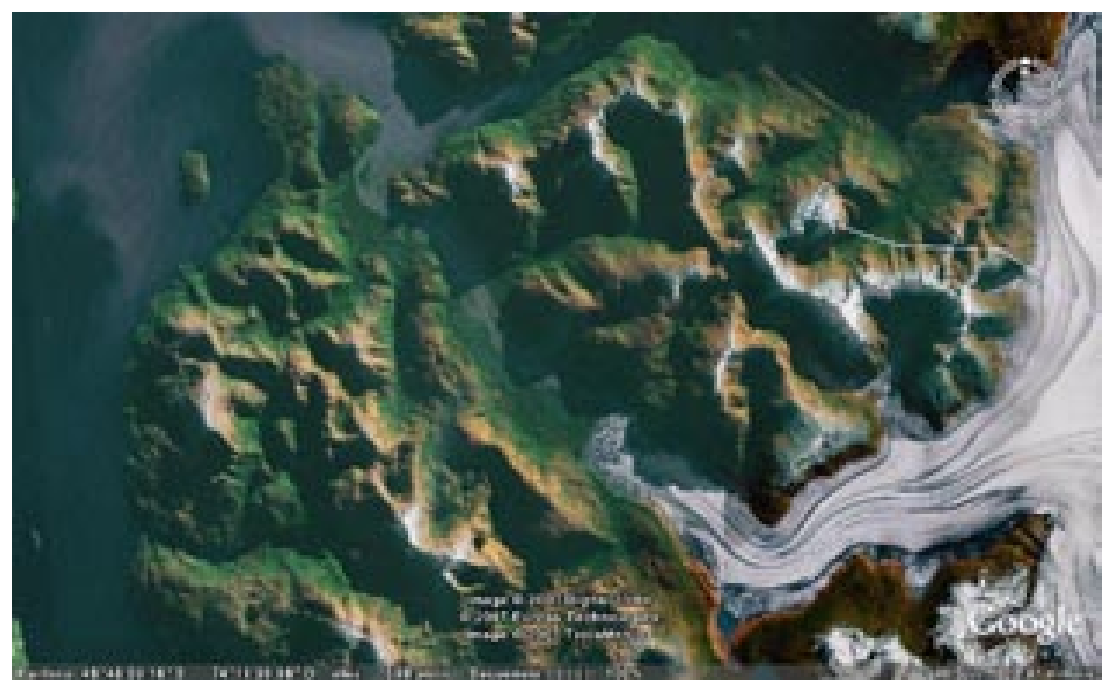

Fig. 1. Glaciar Témpano, valle del río Témpano, (48ํ49’ S - 74ํ12’ W).

El trabajo de campo fue realizado durante los meses de febrero a abril del año 2005 y de octubre a enero del año 2006. La identificación de las especies la realizaron el Dr. Carlos Ramírez, Dr. Juan Marcos Henríquez, Prof. Orlando Dollenz y el Lic. Osvaldo Vidal. Se incluyen en el catálogo tres especies de musgos (Bryophyta) no vasculares.

\footnotetext{
${ }^{1}$ Corporación Nacional Forestal, Magallanes, Chile.

${ }^{2}$ Universidad de Magallanes, Facultad de Ciencias, Dpto. Ciencias y Rec. Naturales.
} 
Las especies herborizadas están depositadas en la Corporación Nacional Forestal, Punta Arenas, región de Magallanes y Antártica Chilena.

\section{CARACTERÍSTICAS GEOGRÁFICAS DEL VALLE DEL RÍO TÉMPANO.}

Clima. Las características climáticas más ajustadas al valle son las que registra la estación climática más cercana, Puerto Edén (Zamora \& Santana 1979). La suma anual de precipitaciones es de $4.068 \mathrm{~mm}$, distribuidas homogéneamente a lo largo del año. La temperatura media anual es de $7,2 \stackrel{\circ}{\circ}$, la media del mes más frío, julio, $2,8 \stackrel{\circ}{ } \mathrm{C}$ y la media del más cálido, enero, 11,6 ㄷ.

Geomorfología. El valle del río Témpano (Fig.1) está rodeado de cerros rocosos aborregados de hasta $900 \mathrm{~m}$ de altitud. En el extremo cordillerano del valle se encuentra el glaciar Témpano del cual nace el río, que desemboca en el canal Messier. El valle en el curso del río está relleno con material glaciofluvial.

Vegetación. La vegetación que cubre las rocas y los sedimentos glaciofluviales del valle como las comunidades boscosas, arbustivas, turbales y litorales coinciden en gran parte en su composición florística con las descritas por Dollenz (1982) para Puerto Kaiser y fiordo Triple en la cercana isla Wellington ubicada en la orilla opuesta del canal Messier.

Las comunidades vegetales diferenciadas por su fisonomía son:

Pradera alterada por pisoteo y pastoreo de ganado doméstico. Se encuentra en la desembocadura del río y presenta, entre las especies nativas, herbáceas introducidas como Rumex acetosella, Sagina procumbens, Holcus lanatus, Raphanus sativus, Dactylis glomerata,Plantago major, Veronica serpyllifolia, Trifolium repens, Mentha $\mathrm{x}$ piperita.

Zona altoandina con vegetación esparcida de baja cobertura. Especies características son Lycopodium confertum, Bolax caespitosa y Azorella lycopodioides.

Bosque de coihue y canelo de Magallanes. Es un bosque siempreverde dominado por Notho- fagus betuloides y Drimys winteri. Otras especies arbóreas son Pilgerodendron uviferum, Podocarpus nubigenus, Weinmannia trichosperma, Pseudopanax laetevirens, Maytenus magellanica. Especies trepadoras del bosque son Mitraria coccinea, Philesia magellanica, Griselinia ruscifolia, Campsidium valdivianum. Hay numerosas especies de helechos en especial himenofiláceos de los géneros Hymenophyllum, Hymenoglossum, Serpyllopsis. Las hepáticas son abundantes en el sotobosque y los troncos.

Turbales. Los turbales se presentan como parches de esfagno, Sphagnum magellanicum asociado a especies como Sphagnum fimbriatum, Marsippospermum grandiflorum, Empetrum rubrum, Myrteola nummularia, Tetroncium magellanicum, Nanodea muscosa, Perezia lactucoides, Carex magellanica. Otros parches turbosos están dominados por especies acojinadas como Astelia pumila, Caltha dionaeifolia, Donatia fascicularis Oreobolus obtusangulus. En roqueríos turbosos aparecen especies leñosas como Embothrium coccineum, Tepualia stipularis y Lomatia ferruginea y especies herbáceas como Drosera uniflora, Pingüicula antarctica, Senecio trifurcatus y cojines como Bolax caespitosa, Phyllachne uliginosa y Gaimardia australis.

El área periglaciar cercana al hielo presenta junquillares dominados por Marsippospermum grandiflorum, en roqueríos se encuentran Calceolaria tenella, Epilobium ciliatum, Cystopteris fragilis, Baccharis nivalis, una comunidad de matorrales con Ribes magellanicum, Gaultheria mucronata, Berberis ilicifolia, Fuchsia magellanica, Berberis buxifolia, Chiliotrichicum diffusum, Escallonia alpina y pastizales húmedos con Blechnum penna-marina, Gunnera magellanica, Viola magellanica, Acaena magellanica, Symphyotrichum vahlí, Hydrocotyle chamaemorus, Ranunculus biternatus.

Las lagunas presentan especies acuáticas como Potamogeton linguatus, Eleocharis melanostachys, Schoenoplectus californicus, Callitriche antarctica. 


\section{CATÁLOGO DE LAS ESPECIES VASCULARES. Pteridófitas y Espermatófitas.}

* Especies exóticas.

Los números del catálogo identifican los especímenes colectados.

La nomenclatura de las familias sigue a Henríquez et al. (1995) con modificaciones.

\section{PTERIDOPHYTA}

Aspleniaceae

140, 221, Asplenium dareoides Desv.

Blechnaceae

148, 244, Blechnum chilense (Kaulf.) Mett.

146, 246, 342, Blechnum magellanicum (Desv.) Mett.

139, 245, Blechnum penna-marina (Poir.) Kuhn

Dicksoniaceae

134, 327, Lophosoria quadripinnata (J.F.Gmel.) C.Chr.

Dryopteridaceae

142, Polystichum multifidum (Mett.) T. Moore

261, Polystichum plicatum (Poepp. ex Kunze) Hicken

194, Polystichum chilense (H.Christ.) Diels

Gleicheniaceae

144, 202, Gleichenia quadripartita (Poir,) T.Moore

Grammitidaceae

135, 203, Grammitis magellanica Desv.

Hymenophyllaceae

137, 335, Hymenoglossum cruentum (Cav.) C. Presl

102, 103, Hymenophyllum darwinii Hook.f. ex Bosch

100, Hymenophyllum ferrugineum Colla

138, 334, Hymenophyllum pectinatum Cav.

333, Hymenophyllum tortuosum Hook. et Grev.

104, 105, Hymenophyllum secundum Hook. et Grev.

98, 336, Serpyllopsis caespitosa (Gaudich.) C.Chr.

Lycopodiaceae

136, 311, Huperzia fuegiana (Roiv.) Holub

307, 310, Lycopodium confertum Willd.

141, 309, Lycopodium gayanum J.Remy

143, 308, Lycopodium magellanicum (P.Beauv.) Sw.

Ophioglossaceae

145, 209, Ophioglossum crotalophoroides Walter 
Schizaeaceae

222, Schizaea fistulosa Labill.

Woodstaceae

147, 220, Cystopteris fragilis (L.) Bernh. var. apiiformis (Gand.) C.Chr.

\section{GIMNOSPERMAE}

Cupressaceae

178, 287, Pilgerodendron uviferum (D. Don) Florin

Podocarpaceae

179, 192, Lepidothamnus fonkii Phil.

181, 193, Podocarpus nubigenus Lindl.

\section{ANGIOSPERMAE: DICOTYLEDONEAE}

Araliaceae

174, 263, Pseudopanax laetevirens (Gay) Franchet

Berberidaceae

160, 242, Berberis microphylla G. Forst.

161, 162, 241, Berberis ilicifolia L.f.

Bignoniaceae

155, 243, Campsidium valdivianum (Phil.) Skottsb.

Boraginaceae

* 247, Myosotis albiflora Banks et Sol. ex Hook. f.

Callitrichaceae

9, 249, Callitriche antarctica Engelm. ex Hegelm.

Campanulaceae

48, 248, Lobelia oligophylla (Wedd.) Lammers

Caryophyllaceae

* 37, 254, Cerastium fontanum Baumg. ssp. vulgare (Hartmann) Greuter et Burdet

44, 253, Colobanthus quitensis (Kunth) Bartl.

* 39, 251, Sagina procumbens L.

* 17, 250, Spergularia rubra (L.) J. Presl. et C. Presl

* 252, Stellaria media (L.) Cirillo

Celastraceae

182, 255, Maytenus magellanica (Lam.) Hook.f.

Compositae

264, Abrotanella linearifolia A.Gray

7, 283, Baccharis nivalis (Wedd.) Sch. Bip. ex Phil.

167, 282, Baccharis patagonica Hook. et Arn. 
165, 281, Chiliotrichum diffusum (G.Forst.) Kuntze

55, 99, 278, Gamochaeta spiciformis (Sch.Bip.) Cabrera

62, 279, Hypochaeris patagonica Cabrera

52, 277, Lagenophora nudicaulis (Comm. ex Lam.) Dusén

16, 276, Leptinella scariosa Cass.

56, 275, Macrachaenium gracile Hook.f.

26, 274, Perezia lactucoides (Vahl) Less.

34, 273, Perezia magellanica (L. f.) Less.

6, 280, Senecio acanthifolius Hombr. et Jacquinot

59, 272, Senecio smithii DC.

43, 271, Senecio tricuspidatus Hook. et Arn

32, 270, Senecio trifurcatus (G.Forst.) Less. ex DC.

15, 284, Symphyotrichum vahlii (Gaudich.) G.L. Nesom

* 3, Taraxacum officinale Weber ex F.H.Wigg

Cornaceae

153, 285, Griselinia ruscifolia (Clos) Ball

Crassulaceae

286, Crassula moschata G.Forst.

Cruciferae

51, 289, Cardamine glacialis (G.Forst.) DC.

* 1, 288, Raphanus sativus L.

Cunoniaceae

156, 317, Weinmannia trichosperma Cav.

Desfontainiaceae

157, 326, Desfontainia fulgens D.Don

Donatiaceae

14, 195, Donatia fascicularis J.R. Forst. et G.Forst.

Droseraceae

28, 197, Drosera uniflora Willd.

Empetraceae

166, 198, Empetrum rubrum Vahl ex Willd.

Epacridaceae

158, 196, Lebetanthus myrsinites (Lam.) Dusén

Ericaceae

150, 260, 259, 328, Gaultheria antarctica Hook.f.

106, 172, 330, Gaultheria mucronata (L. f.) Hook. et Arn.

258, Gaultheria phillyreifolia (Pers.) Sleumer

149, 329, Gaultheria pumila (L. f.) D.J.Middleton 
Escalloniaceae

168, 232, Escallonia alpina Poepp. ex DC.

169, 233, Escallonia serrata Sm.

Fagaceae

184, 331, Nothofagus antarctica (G.Forst.) Oerst.

173, 332, Nothofagus betuloides (Mirb.) Oerst.

Gentianaceae

200, Gentianella magellanica (Gaudich.) Fabris ex D.M.Moore

Gesneriaceae

154, 201, Mitraria coccinea Cav.

Grossulariaceae

175, 234, Ribes magellanicum Poir.

Gunneraceae

58, 291, Gunnera lobata Hook.f.

53, 292, Gunnera magellanica Lam.

Labiatae

* 205, Mentha x piperita L.

Lentibulariaceae

31, 206, Pingüicula antarctica Vahl

Misodendraceae

152, 208, Misodendrum brachystachyum DC.

Myrtaceae

170, 300, Myrteola nummularia (Poir.) O.Berg.

176, 301, Tepualia stipularis (Hook. et Arn.) Griseb.

Onagraceae

8, 299, 298, Epilobium ciliatum Raf.

40, 297, Epilobium conjungens Skottsb.

164, 296, Fuchsia magellanica Lam.

Oxalidaceae

50, 211, Oxalis magellanica G.Forst.

Papilionaceae

* 4, 212, Trifolium repens L.

Plantaginaceae

11, 312, Plantago barbata G.Forst.

* 2, 313, Plantago major L. 
Polygonaceae

* 54, 302, Rumex acetosella L.

* 5, 262, Rumex obtusifolius L.

Primulaceae

49, 101, 214, Anagallis alternifolia Cav.

Proteaceae

177, 304, Embothrium coccineum J.R. Forst. et G. Forst.

159, 303, Lomatia ferruginea (Cav.) R.Br.

Ranunculaceae

63, 341, Caltha appendiculata Pers.

13, 340, Caltha dionaeifolia Hook.

95, Ranunculus biternatus Sm.

339, Ranunculus hydrophilus Gaudich.

96, Ranunculus aquatilis L.

41, 97, 338, Ranunculus minutiflorus Bertero ex Phil.

38, 337, Ranunculus peduncularis Sm.

Rosaceae

23, 228, Acaena antarctica Hook.f.

20, 229, Acaena magellanica (Lam.) Vahl

22, 230, Acaena ovalifolia Ruiz et Pav.

21, 231, Acaena pumila Vahl

42, 227, Geum involucratum Pers.

57,226 , Rubus radicans Cav.

* 257, Rubus idaeus L.

Rubiaceae

65, 199, Nertera granadensis (Mutis ex L.f.) Druce

Salicaceae

* 151, Salix viminalis L.

Santalaceae

27, 215, Nanodea muscosa Banks ex C.F.Gaertn.

Scrophulariaceae

64, 236, Calceolaria tenella Poepp.

239, Euphrasia antarctica Benth.

238, Hebe elliptica (G.Forst.) Pennell

47, 240, Ourisia ruellioides (L.f.) Kuntze

30, 93, Ourisia breviflora Benth.

* 237, Veronica serpyllifolia L.

Stylidiaceae

216, Phyllachne uliginosa J.R. Forst. et G.Forst. 
Tribelaceae

171, 235, Tribeles australis Phil.

Umbelliferae

19, 189, Apium australe Thouars

46, 188, Azorella lycopodioides Gaudich.

45, 185, Bolax caespitosa Hombr. et Jacquinot

61, 187, Hydrocotyle chamaemorus Cham. et Schltdl.

186, Lilaeopsis macloviana (Gand.) A.W. Hill

18, Schizeilema ranunculus (d'Urv.) Domin

Valerianaceae

33, 217, Valeriana lapathifolia Vahl

Violaceae

36, 94, Viola commersonii DC. ex Ging.

35, 218, Viola magellanica G.Forst.

Winteraceae

183, 219, Drimys winteri J.R. Forst. et G. Forst.

ANGIOSPERMAE: MONOCOTYLEDONEAE

Centrolepidaceae

10, 295, Gaimardia australis Gaudich.

Cyperaceae

89, 125, 133, Carex andersonii Boott.

68, 318, Carex banksii Boott.

74, 325, Carex camptoglochin V.I.Krecz.

81, 324, Carex canescens L.

126, Carex fuscula d'Urv.

122, Carex kingii (Boott) Reznicek

80, 323, Carex magellanica Lam.

75, 119, Carpha schoenoides Banks et Sol. ex Hook. f.

121, 123, 127, Eleocharis albibracteata Nees et Meyen ex Kunth

83, 322, Eleocharis melanostachys (d'Urv.) C.B. Clarke

76, 321, Oreobolus obtusangulus Gaudich.

69, 320, Schoenoplectus californicus (C.A.Mey.) Soják

131, Schoenus antarcticus (Hook. f.) Dusén

70, 120, Schoenus rynchosporoides (Steud.) Kûk.

78, 130, Uncinia macloviana Gaudich.

79, 319, Uncinia tenuis Poepp.ex Kunth

86, 124, Uncinia triquetra Kûk.

Gramineae

92, 109, 118, Agrostis magellanica Lam.

108, Agrostis uliginosa Phil.

107, Agrostis perennans (Walter) Tuck. 
72, Agrostis glabra (J.Presl) Kunth

73, 315, Anthoxanthum redolens (Vahl) P. Royen

87, Bromus coloratus Steud.

290, Cortaderia pilosa (d'Urv.) Hackel

* 66, 316, Dactylis glomerata L.

91, Deschampsia kingii (Hook. f.) E. Desv.

115, Elymus angulatus J. Presl

* 67, 314, Holcus lanatus L.

90, 256, Poa alopecurus (Gaudich.)Kunth ssp. fuegiana (Hook.f.)D.M.Moore et Dogg.

* 113, Poa pratensis L.

117, Poa pungionifolia Speg.

110, Poa sp.

111, Gramineae

112, Agrostis sp.

114, Gramineae

116, Poa sp.

Iridaceae

191, Libertia chilensis (Molina) Gunckel

12, 190, Tapeinia pumila (G. Forst.) Baill.

Juncaceae

88, Juncus procerus E.Mey.

85, 128, 129, Juncus scheuchzerioides Gaudich.

82,132, Luzula excelsa Buchenau

71, 294, Marsippospermum grandiflorum (L.f.) Hook.

84, 293, Rostkovia magellanica (Lam.) Hook.f.

Juncaginaceae

77, 204, Tetroncium magellanicum Willd.

Liliaceae

24, 207, Astelia pumila (G.Forst.) Gaudich.

Orchidaceae

29, 210, Codonorchis lessonii (d'Urv.) Lindl.

Philesiaceae

25, 306, Luzuriaga marginata (Banks et Sol. ex Gaertn.) Benth.

163, 305, Philesia magellanica J. F. Gmel.

Potamogetonaceae

60, 213, Potamogeton linguatus Hagstr.

BRYOPHYTA (musgos)

225, Dendroligotrichum dendroides (Hedw.) Broth.

224, Sphagnum fimbriatum Wilson

223, Sphagnum magellanicum Brid. 


\section{AGRADECIMIENTOS}

Se agradece al Sr. J. Sotomayor, Sr. G. Igor, Sr. I. Avendaño, Sr. J. Paredes y Sr. A. Vargas su colaboración en la colecta de especímenes en el campo. A los revisores anónimos por sus observaciones y la actualización taxonómica de las especies.

\section{LITERATURA CITADA}

Charlín, R. 2006. Confección de herbario en el sector fiordo Témpano, Parque Nacional Bernardo O'Higgins. Informe CONAF : 9 pp.
Dollenz, O. 1982. Estudios fitosociológicos en las Reservas Forestales Alacalufes e isla Riesco. Anales Instituto Patagonia (Chile) 13:161170.

Henríquez, J.M., E. Pisano \& C. Marticorena 1995. Catálogo de la flora vascular de Magallanes, Chile. Anales Instituto Patagonia, Serie Cs. Nat. (Chile) 23:5-30

Zamora, E. \& A. Santana 1979. Características climáticas de la costa occidental de la patagonia entre las latitudes $46^{\circ} 40^{\prime}$ y $56^{\circ}$ 30 ' S. Anales Instituto Patagonia, (Chile) 10:109-144. 